\title{
Analyzing the replay of a great save
}

Peter Slinger MD FRCPC

I

$\mathrm{N}$ Hockey the greatest saves a goalie makes are often due to scoring opportunities which are caused by a preceding breakdown in the play of the defense. Similarly in Anesthesia, often the times that we are forced to shine, to salvage a deteriorating situation, are due in retrospect to minor mistakes or short-cuts that we have taken in established procedures prior to the life-threatening incident. The case report by $\mathrm{Ng}$ et al. in this issue of the Journal is an excellent example of this. ${ }^{1}$

The authors describe a drastic complication which occurred during a right thoracotomy which was being managed with a left-sided double-lumen tube. The authors were faced with a misplaced double-lumen tube, a desaturating patient that could not be ventilated and a chest that was filling up with blood. Some of the surgical steps immediately prior to the incident are not clear from their description. However, the exact details of dire emergencies are often uncertain even after the dust has settled. Nevertheless, presented with this crashing patient, the authors performed admirably.

First, let's look at the save itself. Unable to ventilate via the double-lumen tube and unable to ascertain its position, the surgeon opened the right mainstem bronchus and inserted a small (5.5- $\mathrm{mm}$ internal diameter) single-lumen endotracheal tube across the carina into the left mainstem bronchus for use as an endobronchial tube. This was presumably then connected to an anesthetic circuit (sterile?) which was passed into the open thorax. This allowed for ventilation and protected the non-operated lung from further soiling from the hemorrhage in the right chest. Although the progression of the desaturation was halted, oxygenation remained inadequate, presumably due to obstruction of the left upper lobe by the unmodified endobronchial tube. To relieve the hypoxemia the authors then passed another small endotracheal tube into the right upper lobe bronchus. With the patient stabilized, they were then able to get sur- gical control of the hemorrhage in the chest, clean out the airway and ultimately finish the case with the original double-lumen tube. The patient survived without complication and I feel this case shows remarkable original thinking by the Anesthesia/Surgery team in what must have been a very stressful situation.

The authors go on to describe a modification of an endotracheal tube to increase its margin of safety as an endobronchial tube. This they do by cutting the distal tip into a "bird's mouth" shape which reduces the length of tube distal to the cuff but preserves the inflation channel for the cuff itself. It is not clear if they actually used this modification in the case reported.

But I would like to focus my attention on the breakdowns in patient safety that preceded this great save. First, although the authors checked the position of their double-lumen tube with a fibreoptic bronchoscope in the supine position immediately after intubation, they failed to recheck it with the bronchoscope after turning the patient to the lateral position. Checking immediately after intubation is useful, but checking after the patient is finally positioned for surgery is mandatory. It is impossible to prevent some movement of a double-lumen tube when turning a patient. Medico-legally it may no longer be defensible if a problem arises intraoperatively with a doublelumen tube and a bronchoscope has not been used to correctly verify placement. ${ }^{2}$

Second, when they had problems reinflating the right lung they chose to manipulate the double-lumen tube rather than recheck the position with the bronchoscope. They were concerned that the tube had migrated so far distally the tracheal lumen was in the left mainstem bronchus beyond the carina. Problems reinflating the operative lung are common, due to secretions, atelectasis, etc. The particular malposition that they were worried about is very uncommon. The bronchoscope must be kept immediately available in the operating room during thoracic surgery. Unless

From the Department of Anesthesia, University of Toronto, Toronto, Ontario, Canada.

Address correspondence to: Dr. Peter Slinger, Department of Anesthesia, Toronto General Hospital, 200 Elizabeth Street, Toronto, Ontario M5G 2C4, Canada. Phone: 416-340-5164; Fax: 416-340-3698; E-mail: peter.slinger@uhn.on.ca 
the situation is life-threatening (in which case the bronchial cuff should be deflated and two-lung ventilation resumed), the double-lumen position should be rechecked with a bronchoscope before repositioning the tube when there are problems.

Finally, as the authors found out, once the airway fills with blood the small pediatric-size bronchoscopes that we use to check double-lumen tubes are almost useless. The suction channels on this caliber of bronchoscopes cannot deal with any significant volume of blood or pus.

The authors are to be congratulated on dealing with such a frightening complication with such an innovative solution. I believe this gives us all another option to potentially manage a patient that cannot be ventilated tracheally during a thoracotomy. However, even more instructive than the great save is to appreciate the kind of trouble we can get ourselves into when we let the defense lapse and break from established protocols and don't verify the position of double-lumen tubes in an appropriate fashion.

\section{Analyser la reprise d'un sauvetage spectaculaire}

Au hockey, les arrêts les plus spectaculaires réalisés par un gardien de but pour sauver la partie dépendent souvent des chances de compter résultant de l'échec d'un jeu antérieur de la défense. De même, souvent en anesthésie, les occasions où nous devons briller pour rétablir une situation qui se dégrade relèvent, en rétrospective, d'erreurs mineures ou de raccourcis que nous avons pris en définissant les procédures qui ont précédé l'incident menaçant la vie du malade. Le cas présenté par $\mathrm{Ng}$ et coll. dans le présent numéro du Journal est un excellent exemple de la situation. ${ }^{1}$

Les auteurs décrivent la complication sévère d'une thoracotomie droite où ils se sont servi d'un tube à double lumière gauche. Ils ont dû faire face à un tube à double lumière mal placé, à un patient en désaturation, avec impossibilité de le ventiler et à une cavité thoracique inondée de sang. La description fournie laisse dans l'ombre certaines étapes qui ont immédiatement précédé l'incident. Les données exactes sur des situations d'urgence sont souvent incomplètes même après que tout est revenu à la normale. Néanmoins, les auteurs ont admirablement réussi à sauver le patient très mal en point.
Examinons d'abord le sauvetage lui-même. Incapable de ventiler par le tube à double lumière et d'établir sa position, le chirurgien a ouvert la bronche souche droite et a inséré un petit tube endotrachéal monolumière de $5,5 \mathrm{~mm}$ de diamètre interne dans la bronche souche gauche, en croisant la carène, et l'a utilisé comme un tube endobronchique. Le tube a probablement été branché à un circuit anesthésique (stérile?), lequel a été passé dans le thorax ouvert. Ce qui a permis la ventilation et a protégé le poumon non opéré d'une contamination plus importante par l'hémorragie originant de l'hémithorax droit. Même si la progression de la désaturation était stoppée, l'oxygénation demeurait inadéquate, probablement à cause de l'obstruction du lobe supérieur gauche par le tube endobronchique non modifié. Pour éliminer l'hypoxémie, les auteurs ont passé un autre petit tube endotrachéal dans la bronche lobaire supérieure droite. Ayant stabilisé l'état du patient, ils ont pu contrôler chirurgicalement l'hémorragie thoracique, rétablir la perméabilité des voies aériennes et, terminer enfin l'opération en utilisant le tube à double lumière original. Le patient a survécu sans séquelles. Ce cas illustre le remarquable esprit d'initiative de l'équipe d'anesthésie et de chirurgie dans une situation certainement très pénible.

Les auteurs décrivent ensuite la modification d'un tube endotrachéal pour accroître la marge de sécurité de ce tube endobronchique. Pour ce faire, ils ont taillé la pointe distale en "bec d'oiseau”, réduisant ainsi la longueur du tube distal au ballonnet et préservant le canal de gonflage du ballonnet lui-même. Il n'est pas clair, toutefois, si cette modification a été effectivement utilisée dans le cas présenté.

Concentrons-nous plutôt sur la dégradation de la sécurité du patient juste avant ce remarquable sauvetage. D'abord, les auteurs ont vérifié la position du tube à double lumière avec un fibroscope bronchique immédiatement après l'intubation alors que le patient était en décubitus dorsal, mais ils n’ont pas revérifié avec le bronchoscope après avoir placé le patient en position latérale. La vérification qui suit immédiatement l'intubation est utile, mais celle qui suit le changement de position définitif, adopté pour l'intervention chirurgicale, est impératif. C'est impossible qu'il n'y ait aucun mouvement du tube à double lumière en tournant le patient. Au plan médico-légal, il pourrait être difficile d'excuser un problème peropératoire avec un tube à double lumière si un bronchoscope n'a pas été utilisé pour vérifier correctement la position du tube. ${ }^{2}$

Ensuite, devant la difficulté de la réinsufflation du poumon droit, ils ont choisi de manipuler le tube à 
double lumière plutôt que de vérifier de nouveau sa position avec le bronchoscope. Ils se sont inquiétés de la possibilité que le tube se soit déplacé distalement, assez loin pour que la lumière du tube se retrouve dans la bronche souche au delà de la carène. Les difficultés de réinsufflation du poumon opéré sont fréquentes et causées par des sécrétions, de l'atélectasie, etc. La mauvaise position qu'ils ont envisagée est pourtant très rare. Le bronchoscope doit être gardé à portée de la main dans la salle d'opération pendant une intervention thoracique. À moins que la situation ne mette la vie du patient en danger, auquel cas le ballonnet bronchique doit être dégonflé et la ventilation bilatérale rétablie, la position du tube à double lumière doit être revérifiée avec un bronchoscope avant de replacer le tube à la suite de difficultés.

Finalement, comme l'ont découvert les auteurs, une fois que les voies aériennes se remplissent de sang, les bronchoscopes pédiatriques de petite taille, utilisés pour vérifier les tubes à double lumière, sont pratiquement inutiles. Les canaux d'aspiration des bronchoscopes de ce calibre ne suffisent pas en présence de tout volume de sang ou de pus significatif.

On doit reconnaître que les auteurs ont trouvé une solution novatrice de faire face à une complication assez angoissante. Cette situation nous fournit une nouvelle possibilité de traitement d'un patient qui ne peut être ventilé par voie trachéale pendant une thoracotomie. Cependant, plus instructif que ce sauvetage spectaculaire, nous prenons conscience du genre de difficulté à laquelle nous pourrions être confrontés si nous sommes moins vigilants et ne suivons pas les protocoles établis et ne vérifions pas la position des tubes à double lumière de façon appropriée.

\section{References}

1 Ng YT, Chung PCH, Hsieh JR, Yu CC, Lau WM, Lin $\Upsilon \mathrm{H}$. Failure to provide adequate one-lung ventilation with a conventional endotracheal tube using a transbronchial approach: a case report. Can J Anesth 2003; 50: 603-6.

2 Pennefather SH, Russel GN. Placement of doublelumen tubes-time to shed light on an old problem (Editorial). Br J Anaesth 2000; 84: 308-10. 